\title{
Natural History of Practice Transformation: Development and Initial Testing of an Outcomes-Based Model
}

\author{
Katrina E. Donabue, $M D, M P H^{1,2}$ \\ Warren P. Newton, MD, MPH ${ }^{1}$ \\ Ann Lefebvre, MSW, $\mathrm{CPHQ}^{1,3}$ \\ Marcus Plescia, MD, MPH \\ ${ }^{1}$ Department of Family Medicine, Univer- \\ sity of North Carolina at Chapel Hill, Cha- \\ pel Hill, North Carolina \\ ${ }^{2}$ Cecil G. Sheps Center for Health Services \\ Research, Chapel Hill, North Carolina \\ ${ }^{3}$ North Carolina Area Health Education \\ Centers, Chapel Hill, North Carolina \\ ${ }^{4}$ North Carolina Division of Public Health, \\ Raleigh, North Carolina
}

Conflicts of interest: authors report none.

\section{CORRESPONDING AUTHOR}

Katrina Donahue, MD, MPH

Department of Family Medicine

University of North Carolina at Chapel Hill

590 Manning Dr

Chapel Hill, NC 27599

kdonahue@med.unc.edu

\begin{abstract}
PURPOSE Practice transformation is the cornerstone of the future of family medicine and health care reform, but little is known about how the process occurs. We sought to develop and test a model of the natural history of practice transformation.

METHODS We developed an outcomes-based model of how a practice moves through practice transformation in 2 phases: (1) initial model created through meetings with collaborative experts and practice facilitators, and (2) clinical and practice systems change reports examined from the first group of participating North Carolina Improving Performance In Practice practices to test and further refine the model.

RESULTS The resultant model described motivators and supports to transformation. Three emerging practice patterns were identified with the model: transformed practices experiencing robust improvement, activated practices with moderate change, and engaged practices with minimal change in measured quality over a 2-year period. Transformed practices showed broad-based improvement; some reached a threshold and others continued to improve. These practices had highly engaged leadership and used data to drive decisions. Activated practices had a slower improvement trajectory, usually encountering a barrier that took time to overcome (eg, extracting population data, spreading practice changes). Engaged practices did not improve or were unable to sustain change; despite good intentions, multiple competing distractions interfered with practice transformation.
\end{abstract}

CONCLUSIONS Practice transformation is a continuous and long-term process. Internal and external practice motivations and specific practice supports provided by a community-based quality improvement program appear to have an impact on engagement, rate of quality improvement, and long-term sustainability. Early successes play a key role as practices learn how to change their performance.

Ann Fam Med 2013;11:212-219. doi:10.1370/afm.1497.

\section{INTRODUCTION}

$\mathrm{P}$ ractice transformation on a large scale is a cornerstone of the future of family medicine and health care reform, but little is known about the natural history of the process. It is clear that office systems changes are necessary for quality improvement $(\mathrm{QI})^{1.5}$ and implementation of the patient-centered medical home (PCMH) ${ }^{6-9}$ Furthermore, leadership, ${ }^{10}$ practice facilitation, ${ }^{11}$ and organizational adaptive reserve ${ }^{12-14}$ modify a practice's ability to transform. To begin to test large-scale interventions to transform practices, however, a more specific description of the process of transformation is necessary.

Prior work suggests that the methods for changing office systems for chronic disease 4 can be applied to other processes of care ${ }^{3}$ in office practices. Edmondson and others have also underscored the importance of 
practices becoming learning organizations; they need to learn how to learn. ${ }^{15,16}$ Successful organizations often experiment with different work flows or office systems and learn from failures. ${ }^{17}$ Finally, on the principle that practice transformation is what practices do, we believe that it is also important to use measured outcomes of care to track the progress of practice transformation.

The North Carolina Improving Performance in Practice program (NC IPIP) provided an opportunity to describe practice transformation from this perspective. Launched in 2005, the goal of NC IPIP is to drive dramatic improvement in care of chronic disease across all primary care practices in the state of North Carolina. ${ }^{18}$ This program strives to improve clinical outcomes with the use of practice facilitators and other interventions to help practices to improve care delivery through changes in office systems. The assumptions of this program are that practices, rather than individuals, are the key units of delivery of primary care, and that office systems drive much of the variance in quality across practices. ${ }^{19}$ This natural experiment in North Carolina was an ideal opportunity to examine the natural history of the transformative process.

We used the first phase of NC IPIP ${ }^{18}$ to develop and test a model of the natural history of practice transformation, giving attention to barriers and facilitators of the process. For the purposes of this report, we developed a provisional outcomes-based definition of transformation: transformed practices are those that demonstrate clinical improvement in at least 3 quality measures over a 2-year period. Qualitatively, they change their culture, make many office system changes, use their own data to drive decision making, improve measures of clinical quality, and strive to continually improve various aspects of their practices.

\section{METHODS}

\section{Setting}

This report examined the first group of practices in the NC IPIP. As described elsewhere, ${ }^{18}$ NC IPIP was led by the American Board of Medical Specialties, cosponsored by the certifying boards and specialty societies of internal medicine, family medicine, and pediatrics, and funded by Robert Wood Johnson Foundation in 2005. During the past 5 years, North Carolina Area Health Education Centers (NC AHEC), in partnership with Community Care of North Carolina, the NC Academy of Family Physicians, the NC Pediatric Society, the NC Division of Public Health, and the major insurers in the state, developed a comprehensive statewide model of community-based practice-level support for QI. Key elements of NC IPIP included statewide common quality measures across insurers, a system for collecting and feeding back quality data to practices, regional quarterly collaborative dinner meetings modeled on the Institute for Healthcare Improvement Breakthrough Series model, ${ }^{1}$ community-based practice facilitators, labeled QI consultants, modest financial support to defray direct costs of participation, and use of continuing medical education and credit for Maintenance of Certification part IV as incentives.

\section{Practices and Data Systems}

The first phase of NC IPIP included 18 primary care practices (family practice, pediatrics, general internal medicine) located in the Mountain and Eastern regions of North Carolina (Table 1). The majority focused on diabetes (13 practices) and used an electronic health record (16 practices). The practices were mixed among private (39\%), public $(34 \%)$, and academic affiliated (27\%).

Table 2 describes the clinical and practice measures available. Practices could focus on diabetes or asthma quality clinical measures. These data were stored centrally and made available to the practice via a collaborative website. QI consultants worked with practices to develop customized interventions, with the overall goal

\section{Table. 1 Description of Practices}

\begin{tabular}{|c|c|}
\hline Characteristic & Value \\
\hline Practices, No. & 18 \\
\hline \multicolumn{2}{|l|}{ Service area, \% } \\
\hline Rural & 33 \\
\hline Urban & 67 \\
\hline \multicolumn{2}{|l|}{ Quality focus, \% } \\
\hline Diabetes & 72 \\
\hline Asthma & 28 \\
\hline \multicolumn{2}{|l|}{ Patients served, No. } \\
\hline Diabetes & 23,500 \\
\hline Asthma & 4,700 \\
\hline \multicolumn{2}{|l|}{ Size, \% } \\
\hline$\geq 5$ Physicians & 56 \\
\hline$<5$ Physicians & 44 \\
\hline \multicolumn{2}{|l|}{ Practice type, \% } \\
\hline Private & 39 \\
\hline Community health center/health department & 34 \\
\hline Academic & 27 \\
\hline \multicolumn{2}{|l|}{ Data extraction capability, \% } \\
\hline Convenience & 17 \\
\hline Random & 44 \\
\hline Total population & 33 \\
\hline Not extracting data & 6 \\
\hline \multicolumn{2}{|l|}{ Practice specialty, \% } \\
\hline Family medicine & 56 \\
\hline Pediatric medicine & 28 \\
\hline Internal medicine & 16 \\
\hline
\end{tabular}




\begin{tabular}{|ll}
\hline Table 2. Data Available in Practices & \\
\hline Clinical Measures & \\
\hline Diabetes improvement over 12 months & Practice Measures (All Practices) \\
Hemoglobin $A_{1 c}>9 \%$ & Practice type \\
Blood pressure $\leq 130 / 80 \mathrm{~mm} \mathrm{Hg}$ & Rural/urban \\
Low-density cholesterol $\leq 100 \mathrm{mg} / \mathrm{dL}$ & Number of physicians \\
Additional measures included: hemoglobin & Specialty \\
$\mathrm{A}_{1 \mathrm{l}} \leq 7 \%$, blood pressure $\leq 140 / 90 \mathrm{~mm}$ & Use of electronic health record \\
Hg, referral for annual eye examination, & Data extraction capability \\
annual eye examination obtained, annual & Prior quality improvement experience \\
influenza shot, annual foot examination, & Quality improvement consultant ratings: \\
low-density cholesterol $\leq 130 \mathrm{mg} / \mathrm{dL}$, & engagement, leadership, registry use, \\
low-density cholesterol measured in past & template use, protocol uses, self-man- \\
year, annual microalbumin measurement, & agement support \\
tobacco counseling, aspirin use & \\
Asthma improvement over 12 months & \\
Asthma control assessed & \\
Use of asthma controller medications & \\
Influenza vaccine & \\
Bundled patient measure of the 3 asthma & \\
measures & \\
Additional measures included: presence of & \\
action plan, tobacco counseling, emer- & \\
gency department use, hospitalization & \\
a Practices chose diabetes or asthma measures. & \\
\end{tabular}

with national organizations; and the fourth author (K.E.D.) brought expertise of leading prevention collaboratives in the state, as well as participating in the evaluation of the state health department collaboratives and the IPIP.

During the first phase, the natural history and key influences on practice transformation were identified by drawing from the Chronic Care Model. ${ }^{22}$ The model was then shared and revised during meetings with national IPIP team members and state leaders who had experience running collaboratives and in monthly discussions with QI consultants working with NC IPIP practices. In the second phase, the authors individually reviewed each practice's monthly diabetes or asthma quality data, concurrent team assessments from the QI consultant, and the previously writ-

of improving measured quality for the whole population of the practice. Emphasis was placed on development of registry functions, standing orders, support for self-management, and fidelity to the program goals, but QI consultants would also work with practices on any practice clinical or business issues the practice identified. In addition to quality data, 2 QI consultants took concurrent notes about the practice goals, leadership, and progress in implementing office systems changes. Finally, QI consultants wrote narrative descriptions of the practice's progress in improving office systems. These data are the basis of this study.

\section{Model Development}

All authors developed and refined the model during multiple meetings over the course of a year. The authors bring extensive experience with practice redesign and transformation various perspectives to the IPIP: one author (A.L.) led the initial NC QI Organization statewide outreach effort to promote electronic health records in private practices (http:// www.thecarolinascenter.org/) and was the original QI consultant in all the IPIP practices and trained the subsequent QI consultants; another (M.P.) brought quality collaborative expertise as a pioneer of the original chronic disease collaboratives in the state ${ }^{5}$; a third author (W.P.N.) led the NC IPIP project ${ }^{18}$ and 2 successful collaboratives of residency practice redesign in the southeast region, ${ }^{20,21}$ and has been extensively involved at the national level with practice redesign ten practice story generated by the lead practice QI consultant (A.L.). The authors deliberated findings as a group and tested the model. The group identified 3 emerging patterns and common themes of success and barriers to transformation among the practices and further refined the model.

The Biomedical Institutional Review Board at the University of North Carolina determined that this study was exempt from the Institutional Review Board approval.

\section{RESULTS}

Model for the Natural History of Transformation Our model for the natural history of transformation is depicted in Figure 1. The initiation phase is driven by both internal and external motivators. Internal motivators include improved reimbursement, increased office efficiencies, better patient outcomes, help meeting the requirements of a federally qualified health center, and often simply "to do the right thing" for the patients. External influences come from such areas as institutional leadership, professional organizations (certifying boards), payer programs (pay-for-performance), and local pressures (practice comparisons). The IPIP program can be understood as influencing the initiation phase in a number of ways, including community meetings of peers supporting transformation and provision of continuing medical education or maintenance of certification part IV credit. 
The intermediate phase included implementing initial system changes, such as a disease registry (standalone or within an electronic health record), templates to guide care, disease-based protocols for the entire treatment team (ie, standing orders), and an effective patient self-management support program. As shown in the model, these system changes help a practice work toward a paradigm change that includes organizational redesign throughout the practice, development of a culture that supports using data to observe the patient population, and a team-based care approach with an emphasis on the patient's self-management of their disease. The model suggests that familiarity with data systems and early successes lead practices to belief and trust in the process and their data, which sustains the practices to the advanced phase and allows for a true practice transformation that sustains the improvements gained in both process and clinical outcomes. In this framework, NC IPIP and other similar programs can support practice transformation by framing organizational learning through data review and bringing learning about best practices from other sites.

\section{Examples}

By examining each of the practices in the NC IPIP program through the use of this model of sustainable practice transformation (Figure 1), participating practices separated into 3 emerging categories: practices experiencing robust improvement over a sustained period of time, practices with moderate change, and practices with minimal change over a period of time. Table 3 describes our practices categorized as engaged, activated, and transformed. Figure 2 gives a pictorial representation of each of these practice types over time.

Transformed practices separated into 2 subgroups, both with substantial broad-based improvement, but one group reached a threshold (subgroup A) and the other continuing to evolve (subgroup B). A key environmental influence for transformed practices was highly engaged organizational leadership. For example, the leadership in one practice showed their support of QI by implementing a peer-review program throughout the organization, where clinicians reviewed and discussed each other's charts as a group as part of the QI initiative. Examples of internal motivators

\section{Figure 1. Framework for sustainable practice transformation.}

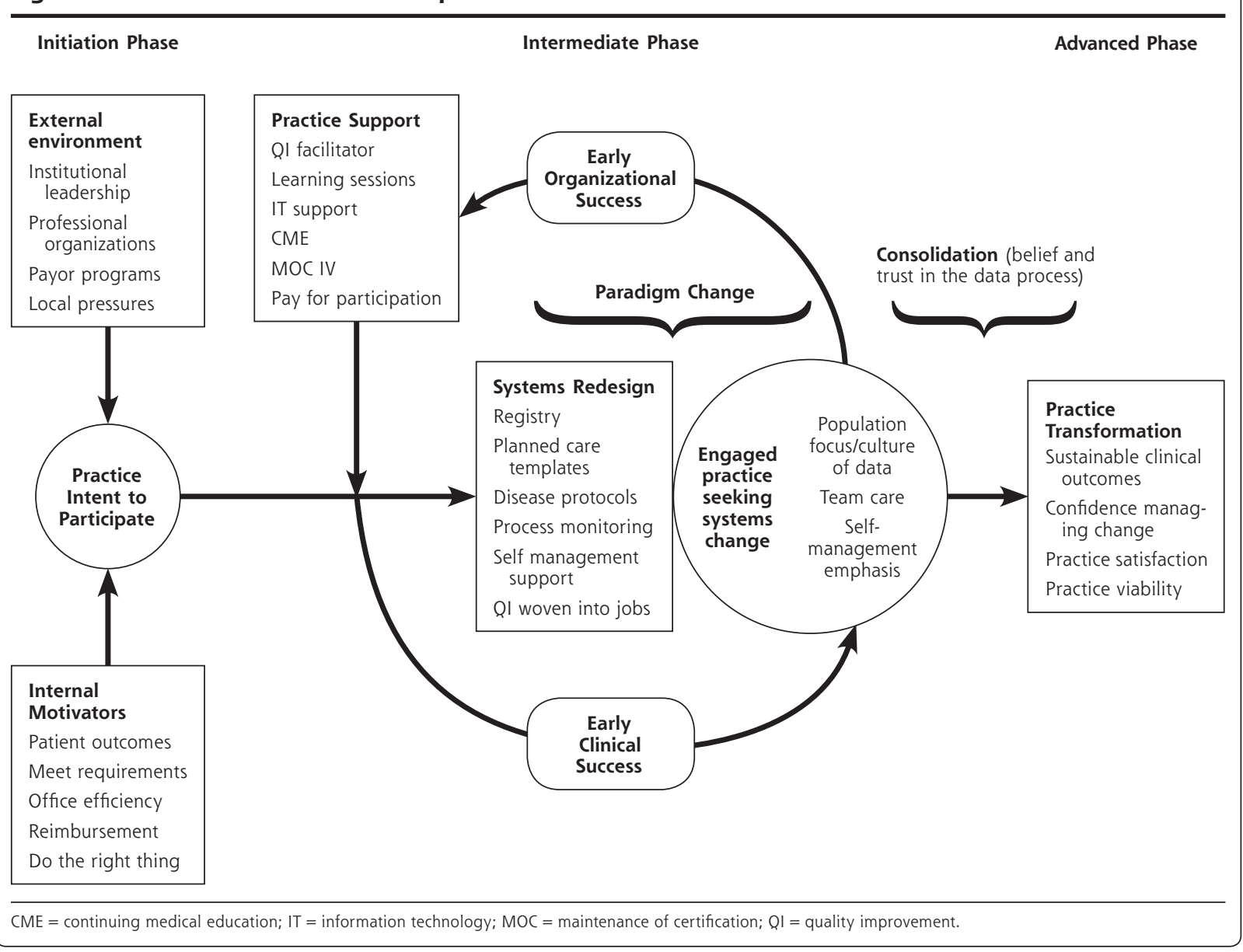


Table 3. Description of Practices by Transformed, Activated, and Engaged

\begin{tabular}{|c|c|c|c|}
\hline & $\begin{array}{l}\text { Transformed } \\
n=7^{\mathrm{a}}\end{array}$ & $\begin{array}{l}\text { Activated } \\
\mathrm{n}=4\end{array}$ & $\begin{array}{c}\text { Engaged } \\
n=7\end{array}$ \\
\hline \multicolumn{4}{|l|}{ Quality focus } \\
\hline Diabetes & 5 & 4 & 4 \\
\hline Asthma & 2 & 0 & 3 \\
\hline \multicolumn{4}{|l|}{ Practice type } \\
\hline Private & 3 & 1 & 3 \\
\hline $\begin{array}{l}\text { Community health center/health } \\
\text { department }\end{array}$ & 3 & 2 & 1 \\
\hline Academic & 1 & 1 & 3 \\
\hline \multicolumn{4}{|l|}{ Practice size } \\
\hline$<5$ Physicians & 5 & 1 & 3 \\
\hline$\geq 5$ Physicians & 2 & 3 & 4 \\
\hline \multicolumn{4}{|l|}{ Location } \\
\hline Rural & 4 & 2 & 2 \\
\hline Urban & 3 & 2 & 5 \\
\hline \multicolumn{4}{|l|}{ Data extraction capability } \\
\hline Electronic, total population & 3 & 1 & 3 \\
\hline Electronic, incremental & 1 & 1 & 2 \\
\hline Random chart audit & 3 & 2 & 2 \\
\hline \multicolumn{4}{|l|}{$\begin{array}{l}\text { Clinical measures improved at } 1 \text { year, }{ }^{b} \\
\text { mean (range), No. }\end{array}$} \\
\hline Diabetes, 14 measures & $6(4-9)$ & $5(5-7)$ & $4(4-5)$ \\
\hline Asthma, 8 measures & $5(4-7)$ & - & $1(0-6)$ \\
\hline \multicolumn{4}{|c|}{$\begin{array}{l}\text { Clinical measures sustained or improved } \\
\text { at year } 2 \text {, mean (range), No. }\end{array}$} \\
\hline Diabetes, 14 measures & $6(3-9)$ & $6(5-7)$ & $4(0-7)$ \\
\hline Asthma, 8 measures & $6(5-7)$ & - & $1(0-3)$ \\
\hline
\end{tabular}

Figure 2. Representation of level of quality over time for transformed practices, activated practices, and engaged practices over 2 years.

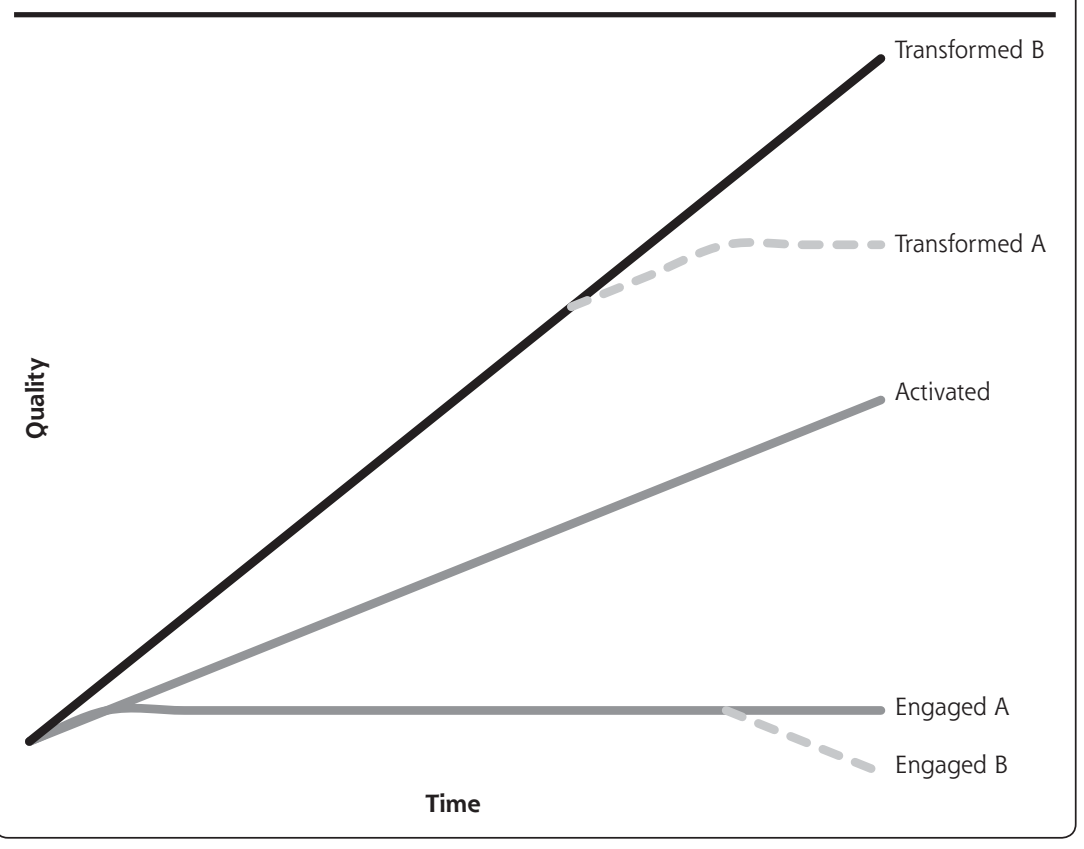

in robust practices included the organizational need to attain federally qualified health center status, which required a QI program. Key practice support elements included active use of the QI consultant on a regular basis for all QI activities. Several transformed practices took advantage of the credit offered for continuing medical education and maintenance of certification. Large multidisciplinary practice teams attended the peer networking meetings and shared regularly at these meetings. The change paradigm for transformed practices included beginning to use their data to drive decision making and change the culture of the organizations. Several robust practices shared physician-level data at all meetings and displayed outcomes data in a public area for patient viewing. For example, practices used data to decide on the specific office systems for redesign and then used the data to monitor their reliability; one practice even planned and measured an intervention to decrease the number of geese crowding the parking lot. Transformed practices also began to include QI activities in job descriptions and compensation plans for staff, thus institutionalizing the practice transformation.

Activated practices included practices that improved in at least one measure with a slower rate of improvement. These practices had a slower trajectory, but eventually reached goals (Figure 2). As with transformed practices, an important environmental influence was leadership supportive of QI work. Internal motivators included physicians on staff who were QI experts. Practice support included the use of the QI consultant in some practice meetings. Compared with transformed practices, however, only the lead 
physician in several of the activated practices attended all peer networking meetings and shared perspectives. In regard to paradigm change, clinical data were incorporated and shared in large QI meetings, and there was some systems redesign, but the impact on or interaction with the staff was minimal. In all activated practices, there were key systems that limited the abilities to perform. For example, implementation of an electronic health record was a common obstacle ${ }_{i}$ compared with transformed practices, activated practices were less able to problem solve issues in this area. Variability in staff engagement was also more common, in part as a result of physician and staff turnover. Another kind of challenge was involvement in too many practice redesign projects, which limited performance in one.

Engaged practices either did not improve in any clinical measure over the first year or improved but did not sustain improvement (Figure 2); as a result, there was no paradigm change during the first year. Despite an initial interest and commitment to the program, the practices were "warming up" and not ready to fully incorporate a sustainable QI program. Causes included staff turnover, other practice priorities, or fiscal stresses. An engaged practice could have positive motivations. For example, in one practice the leadership cited their conviction that QI was the right thing to do for their patients, their professional society's involvement in the program, and their priority for achieving maintenance of certification part IV requirements. This practice was limited, however, in their capacity to make practice changes (eg, the leadership's vision was not shared throughout practice). There were too many distractions in key areas, ranging from business-related issues (keeping the lights on) to the lack of trust in the team care concept (physicians less willing to engage staff in tasks, staff less willing to do things outside a current job description). In regard to practice support, only the lead physician engaged with the QI consultant and usually on an infrequent basis. At this point, the practice was seeking more consultation for practice management and for electronic health records than for QI.

\section{DISCUSSION}

One year after starting in the program, almost $40 \%$ of our initial practices achieved transformation, defined as substantial improvements in 3 or more measures of clinical quality. These practices were characterized by clear motivation, leadership, and a culture that emphasized the use of data to drive decision making. A similar proportion of the practices made no improvement or only transient improvement, whereas $20 \%$ demonstrated slow progress. Key drivers for our practices included variations in internal motivators, the presence of external support, and the use of data.

It is important to keep in mind the context and limitations of this study. First, though comparable with those in many studies of practice transformation, our sample of 18 practices is relatively smalli importantly, they also represent volunteers and early adopters. NC IPIP has now enrolled more than 1,000 practices; the power of specific influences and the natural history of transformation in early adopters may be systematically different from those in later adopters. A second limitation is the clinical quality data, which were collected by the practices themselves for clinical purposes and thus are more vulnerable to bias. We trained practices on the sampling and reliability of their data extraction. A third limitation is the use of a customized intervention and the use of QI consultants for individual practices - the specific interventions varied from practice to practice; this approach, however, has been used in other studies of practice redesign. We also standardized the general approach to the practices, and the change packages QI consultants provided to the practices for specific issues were the same. A final important feature of the setting of this study was the lack of major financial incentives for the physicians to improve quality; even with the passage of health care reform, the same remains true in many settings.

Some will raise the issue of whether it is appropriate to embed clinical quality outcomes in a study of the influences on practices, underscoring the importance and difficulty of the organizational work that must precede and sustain practice transformation. We acknowledge the importance of this organizational work within practices, but we believe that it is also essential to address clinical outcomes. Practice transformation is what practice transformation does-and we must measure it rather than take the word of the physicians. A generation ago, Feinstein showed that including clinical symptoms and signs dramatically improved the predictive power of classification systems for esophageal cancer based on the TNM system. ${ }^{23}$ We believe that the study of the natural history of practice transformation is at a similar stage and could benefit from a focus on the outcomes, eventually, of course, not just measured quality but also patient experience, cost, and practice viability.

\section{Comparative Models of Practice Transformation}

Our model is similar in some respects to that proposed by Cohen et al. ${ }^{24}$ Like that group, we emphasize the role of motivation of key stakeholders, resources for change, external motivators, and the interaction of these influences over time. We emphasize, however, the critical importance of early experiences. As 
described by Edmondson and others, ${ }_{1}^{16,17,25-27}$ organizations must learn how to improve care, and a key mechanism is early success. Many have commented on the importance of leadership and adaptive reserve. ${ }^{13,14,28}$ Our model suggests that early success leads to what might be thought of self-efficacy, in that the practice can change in response to data and urgent concernsthis attribute is a key ingredient of adaptive reserve. Practices are learning organizations, and they must learn how to learn and learn how to manage change. Physician leadership is critical in this realm.

The external environment is also critical. As emphasized by the future of family medicine report, ${ }^{29}$ the environment for primary care is harsh. Over the course of the first 2 years of our study, 3 of our 18 practices reorganized or changed ownership, suggesting the strength of the headwinds facing primary care in our current environment. The community environment can also promote positive change. Our study takes place in the context of a large-scale project to transform practices, in which substantial efforts have been made to provide external support for practice transformation. In addition to QI consultants, practices were given consistent quality measures across insurers, data support, and frequent feedback; furthermore, regional collaboratives provided peer support along with specialty society and certifying board support. Most important are the data and feedback, which played a critical and simplifying role. Relentless focus on data allows a practice to learn and organizes the culture of the practices. Our experiences suggest that as we seek to transform health care, ongoing community level support and infrastructure are essential.

Our results raise a number of important questions about the natural history of practice transformation. A first issue is our understanding of practice transformation. For some writers, practice transformation seems almost like a religious conversion experience: once it happens, the practice continues to improve along all dimensions continuously and forever. Our best performing practices fell into 2 groups. Indeed, some do seem to be improving quality continuously across all fronts. Others, however, reach a threshold and then move on to other diseases or to other aspects of care or office systems. Although continual improvement across all dimensions is an attractive ideal, primary care must manage many chronic diseases, as well as other dimensions of care, including access, prevention, and patient experience. Reaching a threshold, such as the National Committee for Quality Assurance threshold for a specific disease and measure, and then moving on to other problems may be a more realistic goal for most practices. What we emphasize here, however, is that how we conceptualize transformation has major implications for how we promote and sustain practice transformation through public policy and community interventions.

\section{Policy Questions in Practice Transformation}

Public policy makers often imply that practice redesign and systems change are plug and play, a tangible endpoint that can be scheduled precisely. From our data, however, it is clear that practice redesign takes a long time: these practices are 2 years out and counting. Contemporary practices face limited resources and many substantial challenges to change. Other studies of practice redesign have had similar results. ${ }^{30} \mathrm{We}$ also do not know which of our 2 groups - the activated or the transformed-will achieve the most improvement over the long term (eg, 10 years). Will the tortoise or the hare win the race? Finally, although the warmingup practices with limited or no quality improvement may represent those without the leadership, office systems, or incentives to change, they may in fact also be doing important work that is very necessary but has not yet resulted in outcomes improvement.

\section{Role of Patients and Patient Experience}

Our experience so far is that patients' perspectives do not weigh in appreciably as a motivator or external influence on the initiation or the growth of practice transformation. One reason is because our project initially focused on chronic disease, which often does not address patient satisfaction as directly as other practice transformation interventions, such as advanced access. This focus may change with the further evolution of patient-centered medical homes, the more widespread use of measurement of patient satisfaction and patient experience, and the development of patient advisory boards.

Practice transformation is not an overnight sensation; rather, it is a medium- to long-term process that will vary by practice capacity, leadership, community context, and support. Our focus moving forward is now on improving each critical phase: initiation, early success, and then maintenance, even as the dimensions of practice transformation broaden to include patient experience, transitions of care, and cost of care.

To read or post commentaries in response to this article, see it online at http://www.annfammed.org/content/11/3/212.

Key words: primary health care; quality improvement; practice facilitator; coaching

Submitted February 21, 2012; submitted, revised, August 31, 2012; accepted September 19, 2012.

Acknowledgments: Laura G. Brown, MPH, North Carolina Area Health Education Center; Darren Dewalt, MD, MPH, Department of General 
Medicine, University of North Carolina; Cindy Haynes Morgan, North Carolina Division of Public Health, Diabetes Prevention and Control Branch; and Alfred Reid, MA, Department of Family Medicine, University of North Carolina.

\section{References}

1. Institute for Healthcare Improvement. http://www.ihi.org. Accessed Mar 8, 2011.

2. Glasgow RE, Funnell MM, Bonomi AE, Davis C, Beckham V, Wagner EH. Self-management aspects of the improving chronic illness care breakthrough series: implementation with diabetes and heart failure teams. Ann Behav Med. 2002;24(2):80-87.

3. Glasgow RE, Orleans CT, Wagner EH. Does the chronic care model serve also as a template for improving prevention? Milbank $Q$. 2001;79(4):579-612, iv-v.

4. Wagner EH, Austin BT, Davis C, Hindmarsh M, Schaefer J, Bonom A. Improving chronic illness care: translating evidence into action. Health Aff (Millwood). 2001;20(6):64-78.

5. Wang A, Wolf M, Carlyle R, Wilkerson J, Porterfield D, Reaves J. The North Carolina experience with the diabetes health disparities collaboratives. Jt Comm J Qual Saf. 2004;30(7):396-404.

6. National Committee for Quality Assurance. http://www.ncqa.org/ tabid/631/Default.aspx. Accessed Mar 8, 2011.

7. Davis K, Schoenbaum SC, Audet AM. A 2020 vision of patientcentered primary care. J Gen Intern Med. 2005;20(10):953-957.

8. Rosenthal TC. The medical home: growing evidence to support a new approach to primary care. J Am Board Fam Med. 2008;21(5):427-440.

9. Barr M. The Advanced Medical Home: A Patient-Centered, Physician Guided Model Of Health Care. Philadelphia, PA: American College of Physicians; 2006

10. Bray $P$, Cummings DM, Wolf M, Massing MW, Reaves ]. After the collaborative is over: what sustains quality improvement initiatives in primary care practices? Jt Comm J Qual Patient Saf. 2009:35(10): 502-508.

11. Hogg W, Lemelin J, Moroz I, Soto E, Russell G. Improving prevention in primary care: Evaluating the sustainability of outreach facilitation. Can Fam Physician. 2008;54(5):712-720.

12. Miller WL, Crabtree BF, Nutting PA, Stange KC, Jaen CR. Primary care practice development: a relationship-centered approach. Ann Fam Med. 2010;8(Suppl 1):S68-79; S92.

13. Nutting PA, Crabtree BF, Miller WL, Stewart EE, Stange KC, Jaen CR. Journey to the patient-centered medical home: a qualitative analysis of the experiences of practices in the National Demonstration Project. Ann Fam Med. 2010;8(Suppl 1):S45-56; S92.

14. Nutting PA, Crabtree BF, Stewart EE, et al. Effect of facilitation on practice outcomes in the National Demonstration Project model of the patient-centered medical home. Ann Fam Med. 2010;8(Suppl 1): S33-44; 592.
15. Carroll JS, Edmondson AC. Leading organisational learning in health care. Qual Saf Health Care. 2002;11(1):51-56.

16. Edmondson AC. Strategies for learning from failure. Harv Bus Rev. 2001;89no8(April).

17. Bohmer R, Edmondson A. Organizational learning in health care. Health Forum. 2001;March/Apr:32-35.

18. Newton WP, Lefebvre A, Donahue KE, Bacon T, Dobson A. Infrastructure for large-scale quality-improvement projects: early lessons from North Carolina Improving Performance in Practice. J Contin Educ Health Prof. 2010;30(2):106-113.

19. Margolis PA, DeWalt DA, Simon JE, et al. Designing a large-scale multilevel improvement initiative: the improving performance in practice program. J Contin Educ Health Prof. 2010;30(3):187-196.

20. Newton W, Baxley E, Reid A, Stanek M, Robinson M, Weir S. Improving chronic illness care in teaching practices: learnings from the $I^{3}$ collaborative. Fam Med. 2011;43(7):495-502.

21. Reid A, Baxley E, Stanek M, Newton W. Practice transformation in teaching settings: lessons from the $\mathrm{I}^{3} \mathrm{PCMH}$ collaborative. Fam Med. 2011;43(7):487-494

22. Wagner EH. Chronic disease management: what will it take to improve care for chronic illness? Eff Clin Pract. 1998;1(1):2-4.

23. Feinstein A. A new staging system fo rcancer and reappraisal of early treatment and cure by radical surgery. N Engl J Med. 1968; 279:747-753.

24. Cohen D, McDaniel RR Jr, Crabtree BF, et al. A practice change model for quality improvement in primary care practice. $J$ Healthc Manag. 2004;49(3):155-168, discussion 169-170.

25. Thomas P, McDonnell J, McCulloch J, While A, Bosanquet N, Ferlie E. Increasing capacity for innovation in bureaucratic primary care organizations: a whole system participatory action research project. Ann Fam Med. 2005;3(4):312-317.

26. Soubhi H, Bayliss EA, Fortin $M$, et al. Learning and caring in communities of practice: using relationships and collective learning to improve primary care for patients with multimorbidity. Ann Fam Med. 2010;8(2):170-177.

27. Garside P. The learning organisation: a necessary setting for improving care? Qual Health Care. 1999;8(4):211.

28. Stange KC, Miller WL, Nutting PA, Crabtree BF, Stewart EE, Jaen CR Context for understanding the National Demonstration Project and the patient-centered medical home. Ann Fam Med. 2010;8(Suppl 1): S2-8; S92.

29. Martin JC, Avant RF, Bowman MA, et al; Future of Family Medicine Project Leadership Committee. The future of family medicine: a collaborative project of the family medicine community. Ann Fam Med. 2004;2(Suppl 1):S3-S32.

30. Crabtree BF, Nutting PA, Miller WL, Stange KC, Stewart EE, Jaen CR. Summary of the National Demonstration Project and recommendations for the patient-centered medical home. Ann Fam Med. 2010;8(Suppl 1):S80-90; S92. 